Eline Simões Gonçalves a

Renato Marçullo Borges ${ }^{a}$

Leandro Vargas Barreto de Carvalho ${ }^{\text {a }}$

Sérgio Rabello Alves ${ }^{\text {a }}$

Leiliane Coelho André ${ }^{b}$

Josino Costa Moreira ${ }^{a}$

\section{Estratégias analíticas com cromatografia e espectrometria de massas para biomonitorização da exposição ao benzeno pela determinação do ácido $S$-fenilmercaptúrico urinário}

\author{
Analytical strategies with chromatography and mass \\ spectrometry for biomonitoring of benzene exposure by \\ determination of urinary $S$-phenylmercaturic acid
}

\section{Resumo}

Introdução: o benzeno é uma substância de reconhecida toxicidade e sua biomonitorização torna-se fundamental para a prevenção de danos à saúde humana, principalmente em situações de exposição ocupacional. Dentre os biomarcadores de exposição, o ácido $S$-fenilmercaptúrico é considerado o único específico, mas, devido a suas baixas concentrações na urina, é requerido o uso de técnicas analíticas sensíveis capazes de quantificar traços. Objetivo: revisar metodologias baseadas na cromatografia e na espectrometria de massas para a determinação do ácido $S$-fenilmercaptúrico. Método: revisão da literatura sobre a determinação do ácido $S$-fenilmercaptúrico urinário por técnicas de cromatografia e espectrometria de massas, nas principais bases de dados científicas, considerando o período entre 1951 e 2015. Resultados: 120 documentos serviram como base teórica para a construção desta revisão. A técnica analítica mais empregada foi o acoplamento da cromatografia a líquido com a espectrometria de massas. Contudo, os métodos diferem quanto ao preparo das amostras. Conclusão: o alto custo de aquisição e a manutenção de equipamentos são fatores limitantes para a difusão dos sistemas de cromatografia e espectrometria de massas. No entanto, sua elevada sensibilidade e seletividade faz com que essas técnicas, acopladas, possibilitem elucidar situações de exposição ocupacional e ambiental a poluentes, como o benzeno.

Palavras-chave: benzeno; ácido $S$-fenilmercaptúrico urinário; ácido trans, trans-mucônico; cromatografia e espectrometria de massas.

\begin{abstract}
Introduction: benzene is a substance of recognized toxicity and its biomonitoring of exposure becomes critical for preventing health damages, especially in occupational exposure situations. Among all the biomarkers of exposure, $S$-phenylmercapturic acid is considered the only specific one, but due to its low concentrations in urine, acute analytical techniques capable of quantifying traces must be used. Objective: to review methodologies based on chromatography and mass spectrometry for determination of S-phenylmercapturic acid. Method: literature review on the determination of urinary $S$-phenylmercapturic acid by chromatographic techniques and mass spectrometry in the main scientific databases, considering the period between 1951 and 2015. Results: 120 documents served as theoretical basis for the construction of this review. The analytical technique that was most applied was the coupling of liquid chromatography with mass spectrometry. However, the methods differ according to the preparation of samples. Conclusion: the high cost of equipment acquisition and maintenance are limiting factors for the diffusion of chromatography systems and mass spectrometry. Nevertheless, its high sensitivity and selectivity enable these coupled techniques to elucidate situations of occupational and environmental exposure to pollutants, such as benzene.
\end{abstract}

Keywords: benzene; S-phenylmercapturic acid urinary; trans-trans-muconic

acid; chromatography and mass spectrometry.
Recebido: $27 / 10 / 2015$

Revisado: 30/05/2016

Aprovado: 10/06/2016 


\section{Introdução}

Sob o ponto de vista toxicológico, o benzeno é um importante poluente ambiental e, principalmente, ocupacional. É emitido por múltiplas fontes que incluem efluentes industriais, vapores de gasolina, emissões de motores, fumaça do cigarro, dentre outras. Em ambientes de trabalho, sua presença se dá através da emissão de seus vapores ao longo do processo produtivo e de produtos que o utilizam como matéria-prima ou que o têm em sua composição. Um exemplo é o caso dos trabalhadores de postos de revenda de combustíveis (PRC) expostos aos vapores do benzeno provenientes da gasolina $^{1}$.

Desta forma, o benzeno encontra-se amplamente distribuído no ambiente e a exposição a esta substância, seja ambiental ou ocupacional, tem sido associada a sérios danos à saúde humana ${ }^{2}$, tais como o aumento do risco de anemia aplástica, síndromes mielodisplásicas e leucemia mielóide aguda. Desde 1982 é considerado como uma substância comprovadamente carcinogênica para o homem e classificado no grupo A1 pela Agência Internacional para a Pesquisa do Câncer (IARC $^{3-5}$. No Brasil, está inserido no Grupo 1 da Lista Nacional de Agentes Cancerígenos para Humanos (LINACH) .

A avaliação da exposição ao benzeno pode ser realizada por meio da monitorização ambiental, que representa a medida da concentração do benzeno no ambiente (seja em ambientes de trabalho, ou geral); e por meio da monitorização biológica, ou biomonitorização, pela medida da concentração do benzeno e/ou seus produtos de biotransformação em amostras biológicas ${ }^{7}$.

Na avaliação da exposição ocupacional, a concentração deve, preferencialmente, ser determinada na zona respiratória do trabalhador e a medição, realizada com uso de medidores em tempo real ou, como recomendado pela maioria das agências regulatórias, pela coleta de ar por meio de amostradores adequados (ativos ou passivos), utilizando como técnica analítica a cromatografia a gás ${ }^{8}$.

A biomonitorização do benzeno pode ser realizada por meio da medida dos biomarcadores de exposição, os quais podem ser o benzeno no ar expirado, sangue e urina; ou seus metabólitos presentes na urina. Nestes casos, é necessário levar em consideração o tempo em que a exposição aconteceu, devido ao rápido metabolismo e eliminação deste agente tóxico pelo organismo. Em geral, recomenda-se fazer a avaliação em até 24 horas após a exposição ${ }^{3,9}$.

Atualmente existe consenso de que as ações tóxicas do benzeno sobre o organismo humano estão diretamente relacionadas aos seus metabólitos e, assim, a determinação destes compostos tem adquirido especial importância ${ }^{10}$.

A biotransformação do benzeno foi intensamente investigada e sabe-se que em torno de 90\% do benzeno absorvido é excretado através da urina, sob a forma de metabólitos, o que tem justificado a utilização deste tipo de amostra como matriz analítica preferencial. Os metabólitos mais relevantes sob o ponto de vista da quantificação da exposição biológica ao benzeno incluem o ácido $S$-fenilmercaptúrico (AFM), o ácido trans, trans-mucônico (AttM), o fenol (PH), o catecol (CA) e a hidroquinona (HQ) ${ }^{11}$.

Dentre os biomarcadores, o AFM e AttM urinários são os mais utilizados como indicadores de exposição ao benzeno ${ }^{12}$. De modo geral, a determinação do AttM é mais facilmente realizada e, por isto, mais utilizada nos estudos que envolvem este tema, contudo, o AFM é considerado o biomarcador mais sensível e mais específico ${ }^{11,13-18}$.

A concentração urinária do AttM pode ser influenciada pela dieta, uma vez que este biomarcador pode ser originado do metabolismo do sorbitol ou do ácido sórbico, substâncias utilizadas como conservantes de alimentos ${ }^{8,19,20}$. Esta origem limita sua utilização como biomarcador em estudos de saúde pública ${ }^{21}$. Mesmo quando a quantidade de AttM produzida a partir do ácido sórbico não é significativa, ainda assim representa um fator de confundimento na avaliação deste biomarcador em indivíduos expostos a baixos níveis de benzeno e dificulta a interpretação dos dados de grupos de população.

Na biotransformação do benzeno, o AFM é formado a partir de um processo mediado por uma série de enzimas, dentre as quais, as GST (glutationa S-transferases). Alguns estudos têm mostrado que a quantidade de AFM urinário depende de uma série de polimorfismos enzimáticos dos quais sobressaem o CYP2E1, o GSTT1 e o GSTM1. Alguns autores têm encontrado associações entre estes polimorfos e a hematotoxicidade do benzeno ${ }^{22,23}$.

O mecanismo atualmente aceito para a produção do AFM consiste na reação entre o benzeno epóxido, resultante da ação das CYP sobre o benzeno, com a glutationa (GSH), um tripeptídeo (glutamina-cisteína-glicina) que, pela ação da enzima glutationa S-epóxido-transferase, forma o 1-glutationil-2-OH-3,5-ciclo-hexadieno. Este, por sua vez, sofre transpeptidação e perde o resíduo glutamil e, em seguida, por hidrólise, perde o resíduo de glicina. O composto resultante é acetilado pela acetil-coenzima-A a ácido $S$-fenil pré-mercaptúrico (pré-AFM), 
o qual perde uma molécula de água formando o AFM, que é eliminado pela urina ${ }^{24,25}$.

A presença do AFM na urina tem sido associada à concentração do benzeno no ar abaixo de 1 ppm em estudos de exposição ambiental e ocupacional $^{26-28}$. Dessa forma, o uso deste marcador em exposições a baixas concentrações ambientais de benzeno requer técnicas analíticas muito sensíveis capazes de quantificar traços. A principal vantagem do uso do AFM na urina como biomarcador do benzeno é a sua especificidade, já que não sofre influência da dieta ${ }^{17,29}$.

A presença e evidência de um risco químico no ambiente de trabalho podem ser reconhecidas com base na concentração da substância ou seu metabólito no meio biológico e, desta forma, limites biológicos são estabelecidos e/ou recomendados de acordo com as normas de cada país. Devido à dinâmica na produção do conhecimento científico, os marcos regulatórios deveriam ser continuamente atualizados, o que não acontece com a desejada frequência neste país. No Brasil, a Portaria 34 de 20 de dezembro de 2001 do Ministério do Trabalho e Emprego (MTE) estabelece a biomonitorização do benzeno para avaliar uma exposição ocupacional através da determinação de AttM na urina, por este "apresentar características de aplicabilidade, especificidade e sensibilidade para exposição a baixas concentrações de benzeno em ambiente de trabalho" 30 .

No entanto, quinze anos se passaram desde a publicação desta Portaria e, neste intervalo, muitas mudanças foram sugeridas, dentre as quais a utilização do AFM como um biomarcador mais adequado que o AttM. Vários estudos têm investigado a aplicabilidade do AFM como biomarcador de exposição a baixas concentrações de benzeno, e os resultados têm mostrado boa correlação entre os níveis de AFM urinário e o benzeno no $\mathrm{ar}^{20,31-34}$. Atualmente, alguns países já estabelecem o AFM como biomarcador do benzeno nas ações de biomonitorização. Nos Estados Unidos, o NIOSH (National Institute for Occupational Safety and Health) ${ }^{9}$ e a ACGIH (American Conference of Governmental Industrial Hygienists) recomendam o AFM na urina como biomarcador do benzeno, com o limite biológico de exposição de $25 \mu \mathrm{g} \mathrm{g}^{-1}$ de creatinina, cujo valor corresponde a $0,5 \mathrm{ppm}$ de benzeno no $\mathrm{ar}^{35}$.

Entretanto, as limitações associadas ao uso do AFM como biomarcador, especialmente em baixas concentrações do benzeno no ar, estão relacionadas à metodologia analítica, tendo em vista que o AFM representa menos de $1 \%$ do benzeno absorvido e eliminado pela urina. Neste contexto, técnicas cromatográficas combinadas a diferentes detectores têm sido amplamente empregadas para atender a essas exigências de sensibilidade na determinação do AFM urinário.

Assim, este trabalho tem como objetivo apresentar as principais metodologias encontradas na literatura especializada para a determinação do AFM em urina, a fim de servir de orientação para futuros estudos envolvendo o uso deste biomarcador de exposição ao benzeno, principalmente, em estudos de exposição ocupacional em PRC.

\section{Métodos}

Este trabalho é uma revisão bibliográfica realizada entre 2014 e 2015, cujo escopo foi limitado aos estudos que reportam o uso do AFM como biomarcador de exposição ao benzeno por técnicas que envolvam o acoplamento da cromatografia com a espectrometria de massas. Foram consultadas as bases de dados Medline, PudMed e SciELO para a seleção de artigos indexados e também anais de conferências e congressos, capítulos de livros, dissertações e teses disponibilizados na internet e obtidos através das buscas no Google e Google Acadêmico. Foram priorizados os artigos de periódicos que passaram por análise de revisores (peer reviewed) e aqueles provenientes de fontes reconhecidamente confiáveis. O período consultado nas bases bibliográficas abrangeu todos os artigos e trabalhos publicados desde o ano de 1951, quando surgiu o primeiro artigo sobre a presença de AFM em urina.

Os termos-chave (e seus correspondentes em inglês) utilizados nas buscas foram: ácido $S$-fenilmercaptúrico, benzeno, urina, biomarcador, cromatografia, espectrometria de massas. Os artigos foram limitados a textos em português e inglês, e aqueles disponibilizados eletronicamente, na íntegra, no portal de periódicos da Coordenação de Aperfeiçoamento de Pessoal de Nível Superior (Capes).

\section{Resultados e discussão}

Os documentos encontrados foram inicialmente triados analisando-se os títulos e os resumos quanto à realização da determinação do AFM em urina no estudo. A partir disso, os artigos foram selecionados e procedeu-se a uma segunda triagem, em que avaliou-se se havia a descrição da metodologia utilizada para tal análise. Foram selecionados cerca de 120 documentos que serviram como base teórica para a construção deste trabalho. 
Nos documentos selecionados buscou-se informações quanto às principais etapas de desenvolvimento do método analítico (técnicas analíticas; amostragem; armazenamento e estabilidade das amostras; pré-tratamento e procedimentos de extração), que foram usadas como subitens para a apresentação dos resultados encontrados.

\section{Técnicas analíticas}

Diferentes métodos têm sido propostos para determinação do AFM urinário em estudos direcionados à avaliação da exposição humana (ambiental e/ou ocupacional) ao benzeno e seus impactos à saúde $^{8,36-40}$. Alguns estudos considerados relevantes sob o ponto de vista analítico foram compilados neste trabalho, e alguns dos procedimentos utilizados são apresentados na Tabela 1.

Dentre os trabalhos encontrados que utilizam o AFM como biomarcador de exposição ao benzeno, apenas um envolvia trabalhadores de PRC, sendo que o método analítico utilizado foi baseado na técnica de imunoensaio ${ }^{1}$. Entretanto, o ensaio imunoenzimático ELISA (de Enzyme-Linked Immunosorbent Assay) se distingue das técnicas cromatográficas acopladas à espectrometria de massas, principalmente por possuírem menor sensibilidade, ou seja, maiores limites de detecção e de quantificação ${ }^{41-43}$.

De modo geral, os métodos empregados para a determinação do AFM na urina são baseados, principalmente, nas técnicas de cromatografia a líquido (LC) ou a gás (GC) e detecção por espectrometria de massas (MS), sendo que estudos mais recentes recomendam o uso da espectrometria de massas em tan$\operatorname{dem}(\mathrm{MS} / \mathrm{MS})^{18,27,29,44}$. O emprego dessas técnicas se faz necessário devido às baixas concentrações dos biomarcadores normalmente encontrados na urina.

A técnica analítica mais empregada para determinação do AFM é a cromatografia a líquido. Essa técnica oferece vantagens como: o único requisito que a amostra deve apresentar é a solubilidade na fase móvel e grande número de fases estacionárias disponíveis, que proporcionam maior variedade de mecanismos de separação e interação seletiva dos componentes da amostra com as fases cromatográficas ${ }^{45}$. Essas características conferem grande versatilidade à técnica, tornando-a adequada para a separação de inúmeras espécies de interesse, especialmente aquelas não voláteis e termolábeis, como o AFM.

A utilização da cromatografia a gás para a determinação do AFM urinário, apesar de oferecer vantagens analíticas em relação à sensibilidade, alto poder de resolução e menor tempo de análise, é menos aplicada, talvez pela necessidade de derivatizar o AFM a um produto volátil e termoestável ${ }^{46-48}$.

A espectrometria de massas, especialmente quando acoplada à cromatografia a líquido e utilizada em tandem (LC-MS/MS) confere elevada seletividade, sensibilidade e universalidade às análises ${ }^{29}$. O emprego dessa técnica tem simplificado as análises toxicológicas por reduzir o número de etapas no preparo de amostras e com o mínimo de separação cromatográfica. Vários trabalhos são descritos utilizando a cromatografia em fase líquida acoplada ao detector de massas ${ }^{49-51}$. Apesar de todas as vantagens proporcionadas por essa técnica, ela apresenta custo elevado e não elimina completamente o efeito da matriz da amostra, o que leva alguns estudos a utilizar a cromatografia a líquido com detector fluorimétrico, que também apresenta um bom desempenho analítico $^{52,53}$.

O efeito matricial consiste da competição entre componentes da matriz da amostra com o analito(s)-alvo(s) pela ionização, podendo levar a um decréscimo (supressão iônica) ou a um aumento (enriquecimento iônico) da eficiência de ionização do analito-alvo. O efeito matriz pode resultar na diminuição da precisão e acurácia, especialmente em baixas concentrações ${ }^{18,29,54}$.

A resposta do sinal analítico (área e altura do pico cromatográfico), determinada nas amostras reais, deve ser comparada com a resposta do sinal de soluções-padrão de trabalho. No caso do uso de padrão interno deuterado, injeta-se o extrato de urina, dopado com o padrão deuterado e a solução padrão do analito em estudo. Havendo supressão iônica observa-se a presença de picos negativos na linha de base ou menor intensidade do sinal analítico $^{29}$.

A diluição das amostras também pode ser utilizada para minimizar a supressão do sinal causada pela matriz da amostra. Alwis et al. reportam a diluição das amostras de urina na proporção de 1:10 em solução tampão de acetato de amônio ${ }^{20}$. Esses mesmos autores compararam curvas analíticas preparadas com a matriz da amostra (urina) com as preparadas em solução tampão de acetato de amônia de $15 \mathrm{mM}$. Os resultados encontrados mostraram que as inclinações das curvas de calibração nessas duas matrizes foram semelhantes $(p>0,05)$ e as linearidades superiores a 0,99 . Uma revisão interessante sobre os efeitos matriciais em LC-MS/MS foi feita por Tranfo et $\mathrm{al}^{55}$. 


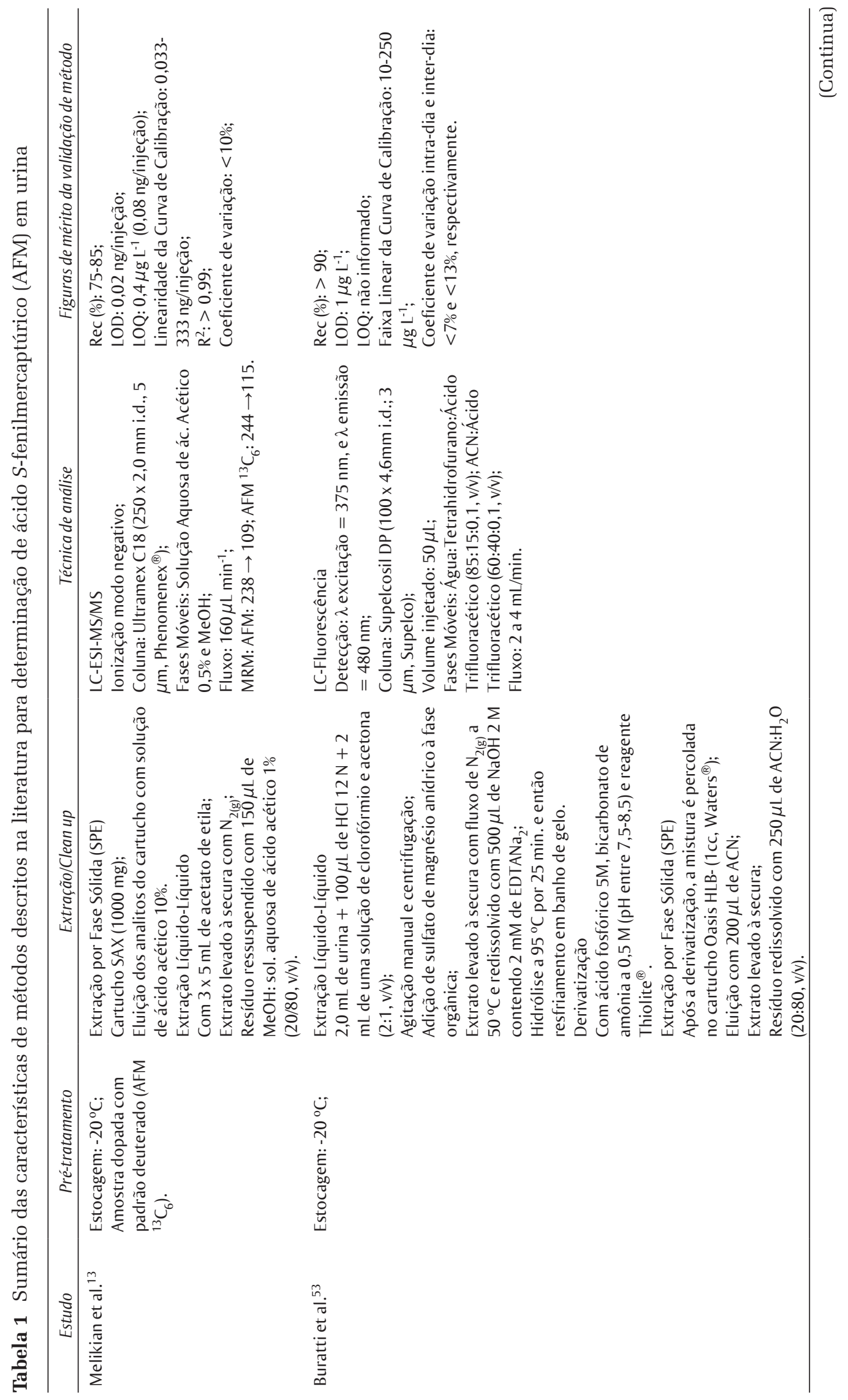




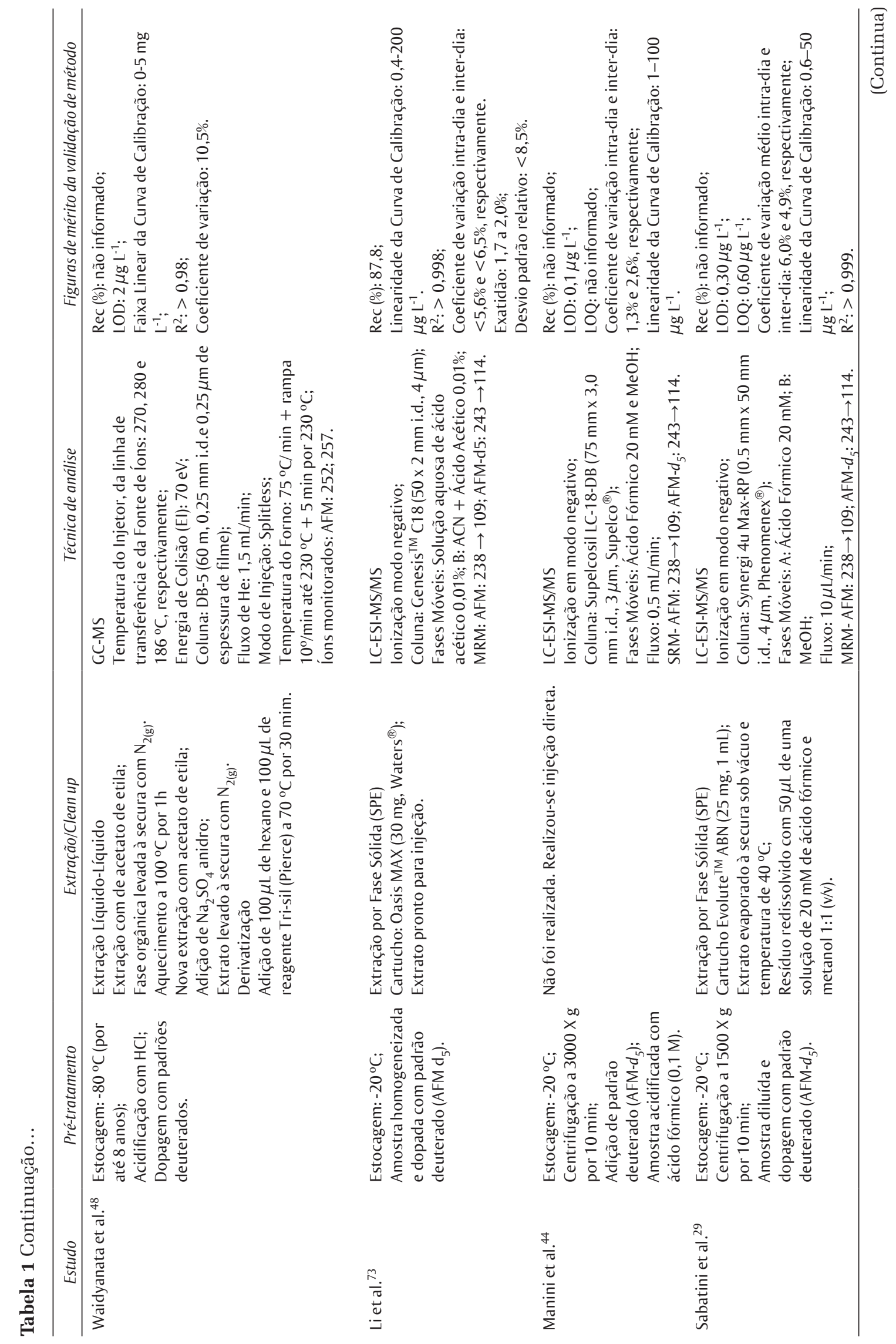




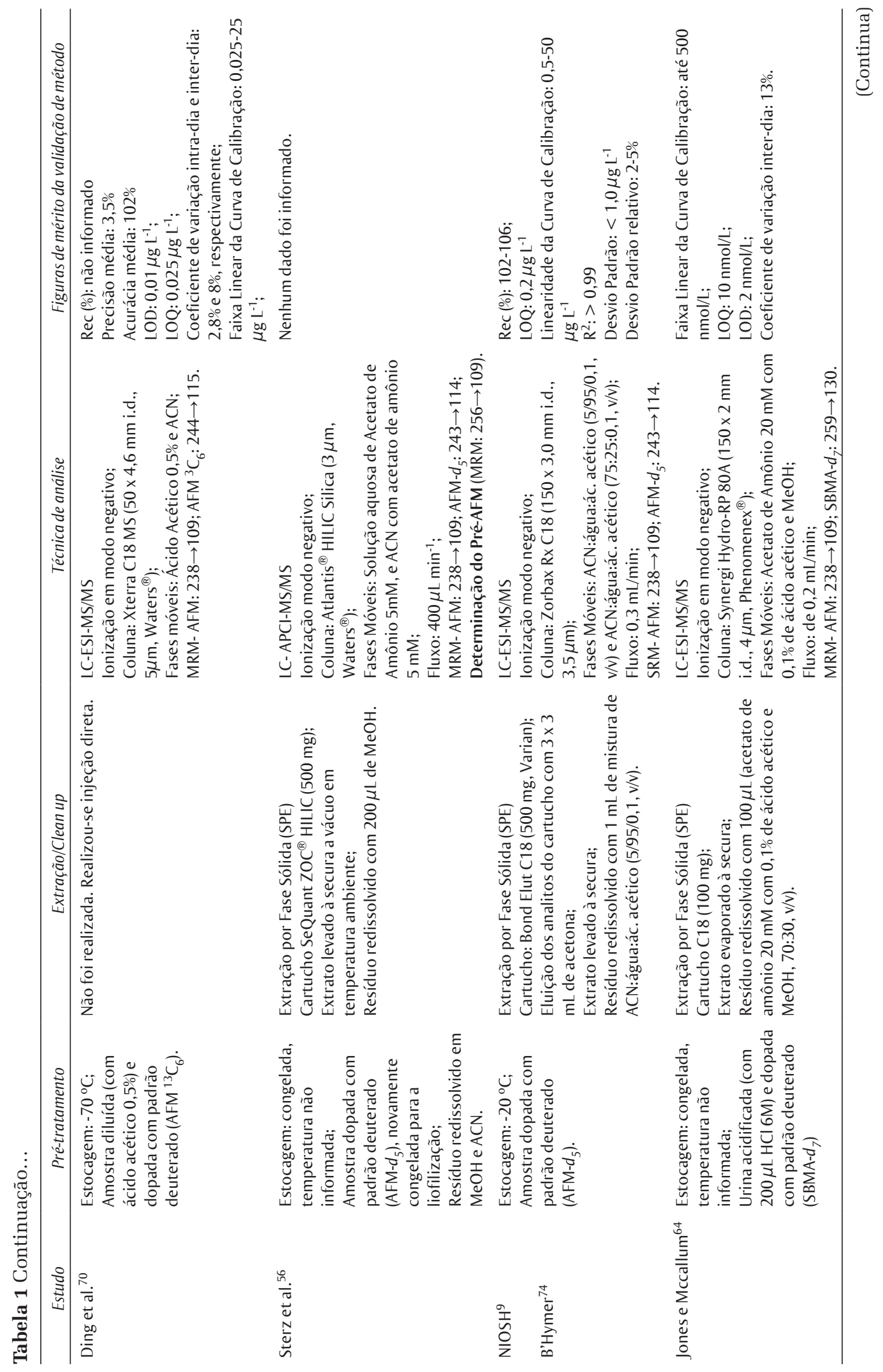




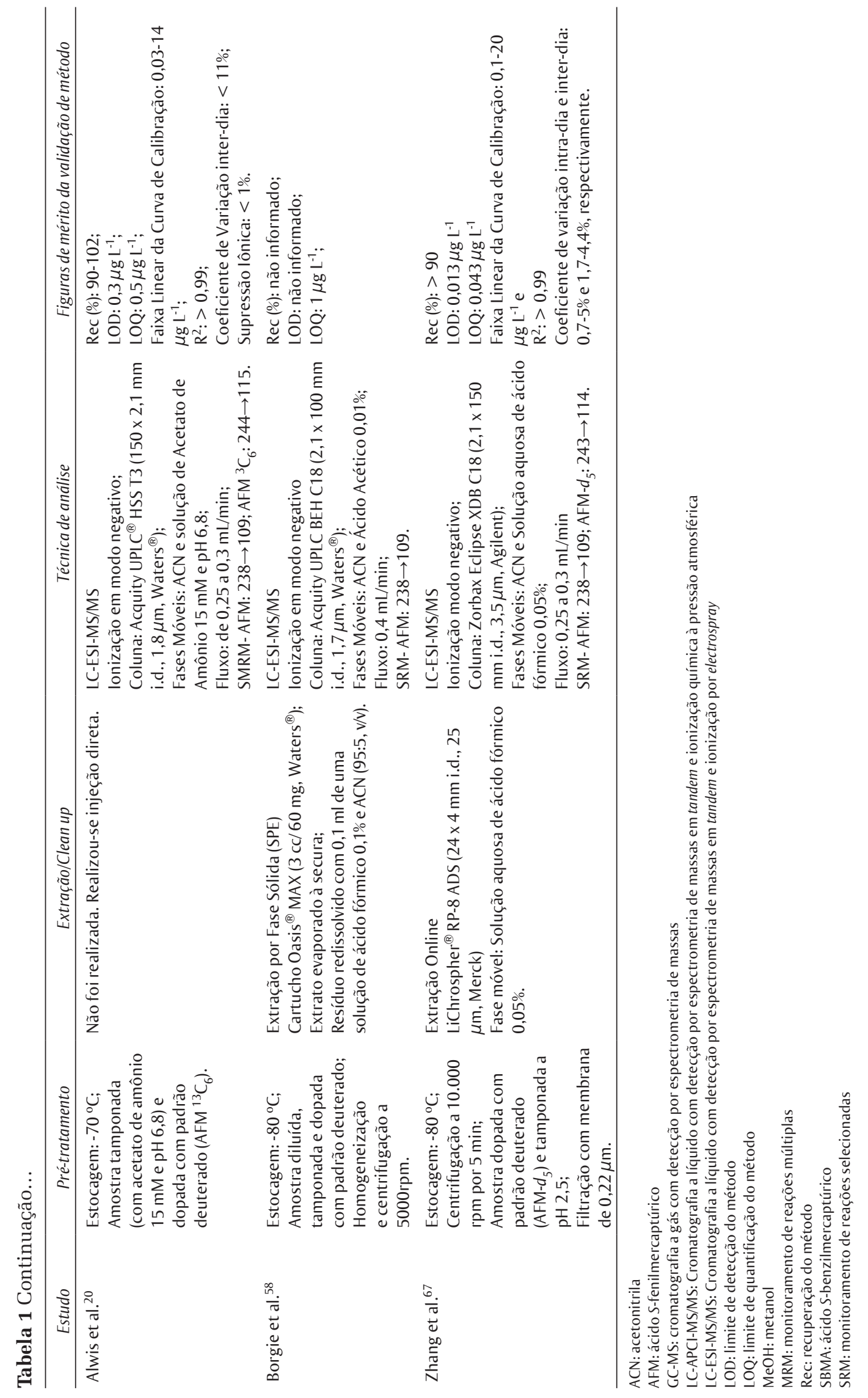


A técnica de ionização comumente utilizada para a análise do AFM por LC-MS/MS é feita pela ionização por electrospray (ESI), que em modo negativo de aquisição gera, a partir do íon molecular de massa-carga (m/z) 238, o íon produto de massa-carga (m/z) $109^{18,29}$. Apenas um estudo foi encontrado utilizando ionização química à pressão atmosférica (APCI de Atmospheric Pressure Chemical Ionization) ${ }^{56}$.

Em geral, a análise por LC-MS/MS, utilizando ESI como fonte de ionização de substâncias contendo grupamento carboxílico em modo negativo, apresentam maior intensidade de sinal quando ionizadas em soluções contendo ácido fórmico em água e/ou metanol ${ }^{29}$.

A utilização da urina como amostra biológica de escolha nas análises de biomarcadores está associada à vantagem de fácil coleta e por ser um método não invasivo. No entanto, como o volume urinário é muito variável e, consequentemente, também a concentração do biomarcador, torna-se necessária uma correção, que é feita através da medida da quantidade de creatinina presente na urina ${ }^{57}$. De acordo com a recomendação da Organização Mundial da Saúde (OMS) para que as amostras de urina sejam aceitáveis para análises de biomarcadores, os níveis de creatinina devem estar entre 0,3 e $3,0 \mathrm{~g} \mathrm{~L}^{-1}$. As limitações desta correção ainda têm sido reportadas ${ }^{32,57,58}$.

Além disso, como a urina é uma amostra biológica complexa, são necessárias outras etapas ao procedimento analítico, como a extração/clean-up e a derivatização aplicadas no preparo da amostra. Desta forma, para o desenvolvimento e/ou adequação de metodologia para a determinação de AFM urinário, é importante que sejam consideradas condições específicas a cada etapa analítica, dentre elas, as mais significativas serão apresentadas a seguir.

\section{Amostragem}

A amostragem é um importante parâmetro no planejamento de estudos epidemiológicos que envolvem a biomonitorização da exposição a substâncias químicas, sendo necessário considerar tanto a toxicocinética quanto a toxicodinâmica da substância no organismo para o planejamento amostral ${ }^{59,60}$.

Estudos estimam a meia de vida de eliminação $\left(t^{1 / 2}\right)$ do AFM e do AttM na urina como de 12,8 e 13,7 $h$, respectivamente ${ }^{61}$. Dessa forma, na avaliação da exposição ao benzeno o AFM irá refletir sempre uma exposição recente. O AFM excretado na urina representa cerca de $0,1 \%$ do benzeno absorvido e sua concentração urinária aumenta continuamente com a exposição, atingindo um máximo cerca de 2 horas após o fim da exposição ${ }^{62}$.
No entanto, uma das preocupações é a influência do tabagismo ativo ou passivo sobre a os biomarcadores de benzeno, já que o fumo do tabaco é uma importante fonte de exposição a esta substância ${ }^{33}$. Para evitar a influência do hábito de fumar sobre os níveis de AFM na urina, Lovreglio et al. sugerem a abstinência de cigarro nas duas horas que precedem a coleta da amostra de urina ${ }^{63}$. Neste contexto, alguns autores realizam a coleta da urina antes do período de exposição, a fim de comparar os níveis iniciais e após a exposição ocupacional ${ }^{64}$.

Para a avaliação da exposição ocupacional, é importante que a coleta da amostra seja realizada ao final da jornada de trabalho. Alguns autores recomendam, inclusive, que a coleta seja feita após, no mínimo, três dias consecutivos de exposição ${ }^{58,65}$.

\section{Armazenamento e estabilidade das amostras}

A integridade de uma amostra biológica está diretamente relacionada à sua estabilidade e consiste em garantir-se que o analito se mantém quimicamente inalterado naquela matriz, sob condições específicas em determinados intervalos de tempo ${ }^{66}$. Em relação ao AFM, são consideradas, principalmente, as condições de armazenamento relacionadas à temperatura, ao tempo de armazenamento (tempo transcorrido entre a coleta e a análise da amostra) e os ciclos de congelamento e descongelamento das amostras ${ }^{67}$.

De modo geral, os estudos indicam que as amostras de urina para a análise do AFM urinário são estáveis nas seguintes condições de armazenamento: temperaturas entre -20 e $-80^{\circ} \mathrm{C}$ e período entre coleta e análise das amostras de 30 a $90 \operatorname{dias}^{20,33}$.

Sabatini et al. realizaram estudos para avaliar a estabilidade do AFM na urina humana em períodos de armazenamento que variaram de 24 horas à temperatura ambiente até 2 meses a $-20^{\circ} \mathrm{C}$, e após 3 ciclos de congelamento e descongelamento ${ }^{29}$. Seus resultados não indicaram diferenças significativas nas concentrações, com variações $\leq 14 \%$. Resultados semelhantes ( $<20 \%$ de variação) foram encontrados por Zhang et al. após três ciclos de congelamento e descongelamento ${ }^{67}$. Outro estudo realizado por Alwis et al. avaliou a estabilidade do AFM e outros metabólitos de Compostos Orgânicos Voláteis (COVs) em amostras mantidas em diferentes temperaturas $\left(22^{\circ} \mathrm{C}, 4^{\circ} \mathrm{C} \mathrm{e}-20^{\circ} \mathrm{C}\right)$ por uma semana e após 10 ciclos de congelamento e descongelamento ${ }^{20}$. De acordo com seus resultados, os autores recomendam que as amostras sejam imediatamente congeladas após a coleta e que até cinco ciclos de congelamento e descongelamento podem ser realizados sem comprometer a estabilidade dos analitos. 
Estudos que relatam a determinação de AFM em amostras de urina armazenadas a longo prazo não são comuns, contudo, Waidyanatha et al. relatam ter analisado suas amostras após 2 e 8 anos de estocagem a $-80^{\circ} \mathrm{C}$. Os autores afirmam que a variabilidade dos resultados foi mínima ${ }^{48}$. Contudo, não são apresentados quaisquer resultados ou discussão sobre a possível degradação das amostras.

Segundo Buratti et al. amostras de urina não tratadas podem ser estocadas a $-18{ }^{\circ} \mathrm{C}$ por pelo menos 6 meses sem qualquer modificação significativa na concentração do $\mathrm{AFM}^{53}$. De acordo com esse estudo, amostras derivatizadas com monobromobimano podem ser estocadas entre $2-8^{\circ} \mathrm{C}$, no escuro, por pelo menos 10 dias sem perda significativa da intensidade do sinal analítico.

Alguns estudos relatam a existência de um precursor do AFM na urina, a $N$-acetil-S-(1,2-dihidro2hidroxifenil)-L-Cisteína, também chamado de pré-AFM, cuja concentração pode variar em função do $\mathrm{pH}$, das condições de estocagem da urina e do tempo de coleta das amostras ${ }^{26,32}$. Desta forma, a fim de diminuir a variabilidade entre os resultados que pode ser causada por diferenças de $\mathrm{pH}$ entre as amostras de urina e também aumentar a concentração de AFM, alguns métodos da literatura recomendam a medida de AFM total, após a transformação do pré-AFM em AFM por hidrólise ácida ${ }^{20,56,68,69}$. Contudo, quando o objetivo do método é uma análise simultânea de outros metabólitos de COVs além do $\mathrm{AFM}^{70,71}$, a hidrólise ácida pode diminuir a estabilidade desses outros analitos ${ }^{20}$.

\section{Pré-tratamento e procedimentos de extração}

A análise de urina para a determinação de biomarcadores como o AFM que, em geral, estão presentes em concentrações-traço (ng L ${ }^{-1}$ ) exige um pré-tratamento da amostra que consiste da eliminação de possíveis interferentes (clean-up) e/ou uma pré-concentração dos analitos-alvo ${ }^{72}$.

Etapas de clean-up/extração são aplicadas para aumentar a sensibilidade analítica, melhorando os limites de detecção e quantificação. No entanto, é mais uma etapa no procedimento analítico e por isso, alguns autores optam por sua não utilização, introduzindo a amostra diretamente no sistema cromatográfico $^{20,44,73}$. Quando isto ocorre, as análises dependem da utilização de equipamentos mais sensíveis, com limites de detecção mais baixos e seletivos ${ }^{20}$.

A extração em fase sólida (SPE, de Solid Phase Extraction) é a técnica mais comumente utilizada para a determinação de AFM urinário. Esta etapa pode ser realizada utilizando diferentes tipos de sorventes para extração, tais como cartuchos de sílica hidrofóbicos (C18, C-fenil, etc.), cartuchos de troca aniônica, ou fase hidrofílica-hidrofóbica balanceada $^{74}$.

A extração de AFM da urina também pode ser feita por extração líquido-líquido ${ }^{54}$. Em geral, essa técnica é realizada em conjunto com a etapa de derivatização do AFM a fim de torná-lo mais volátil para ser analisado por cromatografia a gás.

Apesar das vantagens envolvidas no pré-tratamento das amostras (purificação e concentração), sua realização é laboriosa, e pode trazer maior erro analítico quando comparados a uma injeção direta e automática. Uma metodologia automatizada para a determinação de AFM e outros quatro ácidos mercaptúricos em urina utilizando uma pré-coluna de extração on-line ao sistema LC-MS/MS foi descrita e validada por Zhang et al., apresentando adequada eficiência analítica ${ }^{67}$. Outros estudos utilizando a mesma técnica foram realizados por Liao et al. ${ }^{75}$, Lin et al. ${ }^{76}$ e Schettgen et al. ${ }^{77}$. As desvantagens de extração on-line envolvem a necessidade de aparatos instrumentais específicos que, em geral, devem ser adquiridos à parte da configuração padrão do cromatógrafo e também o custo mais elevado da pré-coluna de extração, quando comparado ao custo dos tradicionais cartuchos de SPE.

\section{Conclusão}

Atualmente, a determinação do AFM tem adquirido grande importância como biomarcador de exposição ao benzeno, devido a sua maior seletividade quando comparada à apresentada pelo marcador usual AttM, que possui maior possibilidade de interferência devido à ingestão de outras substâncias que podem originar sua formação no organismo humano.

A técnica de cromatografia a líquido acoplada à espectrometria de massa em tandem (LC-MS/MS) tem sido proposta para determinação do AFM na urina como biomarcador de exposição ao benzeno, devido a sua elevada sensibilidade, seletividade e amplo intervalo de concentrações. Contudo, o alto custo de aquisição dos equipamentos e sua manutenção, assim como a disponibilidade de profissionais para operacionalização da técnica, são fatores que limitam sua difusão a todos os Laboratórios Centrais de Saúde Pública (Lacen) e, por consequência, limitam a realização de análises deste biomarcador na urina dos trabalhadores expostos ocupacionalmente ao benzeno.

Entretanto, a superioridade da LC-MS/MS sobre as demais, bem como a sua ampla aplicabilidade, não apenas nos estudos que envolvam a determinação da contaminação humana por concentrações 
relativamente baixas de benzeno, mas também a outras substâncias, justifica plenamente sua recomendação como técnica de escolha e, consequentemente, requer sua divulgação. Adicionalmente, a adoção de uma técnica analítica única pelos grupos participantes deste tipo de estudo trará muito mais confiança à comparação dos resultados obtidos.

A evolução das ferramentas analíticas para determinação do AFM na urina permite a quantificação em baixas concentrações, as quais são evidenciadas pelos desempenhos dos métodos analíticos com limites de detecção extremamente baixos. No entanto, outros desafios aos estudos epidemiológicos ainda são colocados, tais como o estilo de vida e fatores genéticos que podem afetar as concentrações dos biomarcadores de exposição ao benzeno, como o AFM e ainda mais o AttM. Portanto, como recomendação, estes fatores devem ser considerados nos estudos com essa finalidade.

\section{Contribuições de autoria}

Todos os autores contribuíram igualmente na pesquisa e construção deste texto.

\section{Referências}

1. Carrieri M, Bonfiglio E, Scapellato ML, Maccà I, Tranfo G, Faranda P, Paci E, Bartolucci G. Comparison of exposure assessment methods in occupational exposure to benzene in gasoline filling-station attendants. Toxicol Lett. 2006;162(2-3):146-52.

2. Bahadar H, Mostafalou S, Abdollahi M. Current understandings and perspectives on non-cancer health effects of benzene: a global concern. Toxicol Appl Pharmacol. 2014;276(2):83-94.

3. Wilbur SB, Keith, Sam MS, Faroon O, Wohlers D. Toxicological profile for benzene [Internet]. U. S. Department of Health and Human Services. Public Health Service. Agency for Toxic Substances and Disease Registry. Atlanta; 2007. Available from: http://bit.ly/2lBf1vV

4. World Health Organization. Biological monitoring of chemical exposure in the workplace. Guidelines. Vol 1. Geneva: WHO; 1996.

5. International Agency for Research on Cancer. IARC Monographs on the evaluation of carcinogenic risks in humans. Dry cleaning, some chlorinated solvents and other industrial chemicals. Geneva: WHO; 1995;63:393-407.

6. BRASIL. Portaria Interministerial MS/MTE n. 9, de 7 de outubro de 2014. Brasil; 2014.

7. Ong CN, Lee BL. Determination of benzene and its metabolites: application in biological monitoring of environmental and occupational exposure to benzene. J Chromatogr B Biomed Appl. 1994;660(1):1-22.

8. Weisel CP. Benzene exposure: An overview of monitoring methods and their findings. Chem Biol Interact. 2010;184(1-2):58-66.

9. National Institute for Occupational Safety and Health. S-Benzylmercapturic acid and S-phenylmercapturic acid in urine Metabolites of toluene and benzene [Internet]. Atlanta: Centers for Disease Control and Prevention; 2014. Available from: http://bit.ly/2mChZ2S
10. Snyder R. Recent developments in the understanding of benzene toxicity and leukemogenesis. Drug Chem Toxicol. 2000;23(1):13-25.

11. Boogaard PJ. Biomonitoring of the Workplace and Environment. In: General, applied and systems toxicology. New Jersey: Wiley; 2009. p. 2559-89.

12. Pieri M, Miraglia N, Acampora A, Genovese G, Soleo L, Sannolo N. Determination of urinary S-phenylmercapturic acid by liquid chromatography-tandem mass spectrometry. J Chromatogr B Analyt Technol Biomed Life Sci. 2003;795(2):347-54.

13. Melikian AA, O'Connor R, Prahalad AK, Hu P, Li $\mathrm{H}$, Kagan M, et al. Determination of the urinary benzene metabolites S-phenylmercapturic acid and trans,trans-muconic acid by liquid chromatography-tandem mass spectrometry. Carcinogenesis. 1999;20(4):719-26.

14. Boogaard PJ, van Sittert NJ. Biological monitoring of exposure to benzene: a comparison between S-phenylmercapturic acid, trans,trans-muconic acid, and phenol. Occup Environ Med. 1995;52(9):611-20.

15. Melikian AA, Qu Q, Shore R, Li G, Li H, Jin X, Cohen L, Li Y, Yin S, Mu R, Zhang X, Wang Y. Personal exposure to different levels of benzene and its relationships to the urinary metabolites S-phenylmercapturic acid and trans, transmuconic acid. J Chromatogr B Analyt Biomed Life Sci. 2002;778(1-2):211-21.

16. Sørensen M, Skov H, Autrup H, Hertel O, Loft S. Urban benzene exposure and oxidative DNA damage: influence of genetic polymorphisms in metabolism genes. Sci Total Environ. 2003;309(13):69-80.

17. Farmer PB, Kaur B, Roach J, Levy L, Consonni D, Bertazzi PA, Pesatori A, Fustinoni S, Buratti M, Bonzini M, Colombi A, Popov T, Cavallo D, 
Desideri A, Valerio F, Pala M, Bolognesi C, Merlo F. The use of S-phenylmercapturic acid as a biomarker in molecular epidemiology studies of benzene. Chem Biol Interact. 2005;153-154:97-102.

18. Manini P, Andreoli R, Mutti A. Application of liquid chromatography-mass spectrometry to biomonitoring of exposure to industrial chemicals. Toxicol Lett. 2006;162(2-3):202-10.

19. Hoet P, De Smedt E, Ferrari M, Imbriani M, Maestri L, Negri S, De Wilde P, Lison D, HAufroid V. Evaluation of urinary biomarkers of exposure to benzene: correlation with blood benzene and influence of confounding factors. Int Arch Occup Environ Health. 2009;82(8):985-95.

20. Alwis KU, Blount BC, Britt AS, Patel D, Ashley DL. Simultaneous analysis of 28 urinary VOC metabolites using ultra high performance liquid chromatography coupled with electrospray ionization tandem mass spectrometry (UPLC-ESI/ MSMS). Anal Chim Acta. 2012;750:152-60.

21. Johnson ES, Langård S, Lin YS. A critique of benzene exposure in the general population. Sci Total Environ. 2007;374(2-3):183-98.

22. Kim S, Lan Q, Waidyanatha S, Chanock S, Johnson B a, Vermeulen R, Smith MT, Zhang L, Li G, Shen M, Yin S, Rothman N, Rappaport SM. Genetic polymorphisms and benzene metabolism in humans exposed to a wide range of air concentrations. Pharmacogenet Genomics. 2007;17(10):789-801.

23. De Palma G, Manno M. Metabolic polymorphisms and biomarkers of effect in the biomonitoring of occupational exposure to low-levels of benzene: state of the art. Toxicol Lett. 2014;231(2):194-204.

24. Snyder R, Hedli CC. An overview of benzene metabolism. Environ Health Perspect. 1996;104Suppl 6:1165-71.

25. Parke D V, Williams RT. Studies in detoxication. 38. The metabolism of benzene: the determination of phenylmercapturic acid in urine; mercapturic acid excretion by rabbits receiving benzene. Biochem J. 1951;48(5):624-9.

26. Tuakuila J. S-phenylmercapturic acid (S-PMA) levels in urine as an indicator of exposure to benzene in the Kinshasa population. Int J Hyg Environ Health. 2013;216(4):494-8.

27. Barbieri A, Violante FS, Sabatini L, Graziosi F, Mattioli S. Urinary biomarkers and low-level environmental benzene concentration: assessing occupational and general exposure. Chemosphere. 2008;74(1):64-9.

28. Bono R, Traversi D, Maestri L, Schilirò T, Ghittori S, Baiocchi C, Gilli G. Urban air and tobacco smoke in benzene exposure in a cohort of traffic policemen. Chem Biol Interact. 2005;153154:239-42.

29. Sabatini L, Barbieri A, Indiveri P, Mattioli S, Violante FS. Validation of an HPLC-MS/MS method for the simultaneous determination of phenylmercapturic acid, benzylmercapturic acid and o-methylbenzyl mercapturic acid in urine as biomarkers of exposure to benzene, toluene and xylenes. J Chromatogr B Analyt Technol Biomed Life Sci. 2008;863(1):115-22.

30. Arcuri ASA, Cardoso LMN. Acordo e Legislação sobre o Benzeno: 10 anos. São Paulo: Fundacentro; 2005.

31. Arayasiri M, Mahidol C, Navasumrit P, Autrup $\mathrm{H}$, Ruchirawat M. Biomonitoring of benzene and 1,3-butadiene exposure and early biological effects in traffic policemen. Sci Total Environ. 2010;408(20):4855-62.

32. Carrieri M, Tranfo G, Pigini D, Paci E, Salamon F, Scapellato ML, Fracasso ME, Manno M, Bartolucci GB. Correlation between environmental and biological monitoring of exposure to benzene in petrochemical industry operators. Toxicol Lett. 2010;192(1):17-21.

33. Protano C, Andreoli R, Manini P, Vitali M. Urinary trans, trans-muconic acid and S-phenylmercapturic acid are indicative of exposure to urban benzene pollution during childhood. Sci Total Environ. 2012;435-436:115-23.

34. Smith MT, Rothman N. Biomarkers in the Molecular Epidemiology of Benzene-Exposed Workers. J Toxicol Environ Health A. 2000;61(56):439-45.

35. American Conference of Governmental Industrial Hygienists. TLVs and BEIs: Threshold Limit Values for Chemical Substances and Physical Agents and Biological Exposure Indices. Cincinnati, Ohio: ACGIH; 2014. 254 p.

36. Coutrim MX, Carvalho LRF, Arcuri ASA. A avaliação dos métodos analíticos para a determinação de metabólitos do benzeno como potenciais biomarcadores de exposição humano ao benzeno no ar. Quim Nova. 2000;23(5):653-63.

37. Perbellini L, Veronese N, Princivalle A. Mercapturic acids in the biological monitoring of occupational exposure to chemicals. J Chromatogr B Analyt Technol Biomed Life Sci. 2002;781(12):269-90.

38. Khan HA. A Concise Review of chromatographic methods for the analysis of benzene and its metabolites. Croat Chem Acta. 2006;79(2):169-75.

39. Arnold SM, Angerer J, Boogaard PJ, Hughes MF, O'Lone RB, Robison SH, Schnatter AR. The use of biomonitoring data in exposure and human health risk assessment: benzene case study. Crit Rev Toxicol. 2013;43(2):119-53.

40. Mathias PI, B’Hymer C. A survey of liquid chromatographic-mass spectrometric analysis of mercapturic acid biomarkers in occupational and environmental exposure monitoring. J Chromatogr B Analyt Technol Biomed Life Sci. 2014;964:136-45.

41. Lohse C, Jaeger LL, Staimer N, Sanborn JR, Jones AD, Langó J, Gee SJ, Hammock BD. Development of a class-selective enzyme-linked immunosorbent assay for mercapturic acids in human urine. J Agric Food Chem. 2000;48(12):5913-23. 
42. Pople JE, Ball RL, Padgett MJ, Aston JP. Construction of a database of benzene biological monitoring. Toxicol Lett. 2002;134(1-3):301-4.

43. Seow WJ, Pesatori AC, Dimont E, Farmer PB, Albetti B, Ettinger AS, Bollati V, Bolognesi C, Roggieri P, Panev TI, Georgieva T, Merlo DF, Bertazzi PA, Baccarelli AA. Urinary Benzene Biomarkers and DNA Methylation in Bulgarian Petrochemical Workers: Study Findings and Comparison of Linear and Beta Regression Models. PLoS One. 2012;7(12):e50471.

44. Manini P, De Palma G, Andreoli R, Poli D, Petyx M, Corradi M, Mutti A, Apostoli P. Biological monitoring of low benzene exposure in Italian traffic policemen. Toxicol Lett. 2008;181(1):25-30.

45. 45. Skoog DA, Holler FJ, Nieman TA. Princípios de Análise Instrumental. 5. ed. Porto Alegre: Bookman; 2002. 836 p.

46. Angerer J, Schildbach M, Krämer A. Gas chromatographic method for the simultaneous determination of S-p-toluylmercapturic acid and S-phenylmercapturic acid in human urine. J Anal Toxicol. 1998;22(3):211-4.

47. Fang MZ, Shin MK, Park KW, Kim YS, Lee JW, Cho $\mathrm{MH}$. Analysis of urinary S-phenylmercapturic acid and trans,trans-muconic acid as exposure biomarkers of benzene in petrochemical and industrial areas of Korea. Scand J Work Environ Health. 2000;26(1):62-6.

48. Waidyanatha S, Rothman N, Li G, Smith MT, Yin S, Rappaport SM. Rapid determination of six urinary benzene metabolites in occupationally exposed and unexposed subjects. Anal Biochem. 2004;327(2):184-99.

49. Fustinoni S, Campo L, Mercadante R, Manini P. Methodological issues in the biological monitoring of urinary benzene and S-phenylmercapturic acid at low exposure levels. J Chromatogr B Analyt Technol Biomed Life Sci. 2010;878(27):2534-40.

50. Maestri L, Negri S, Ferrari M, Ghittori S, Imbriani M. Determination of urinary S-phenylmercapturic acid, a specific metabolite of benzene, by liquid chromatography/single quadrupole mass spectrometry. Rapid Commun Mass Spectrom. 2005;19(9):1139-44.

51. Chiang WC, Chen CY, Lee TC, Lee HL, Lin YW. Fast and simple screening for the simultaneous analysis of seven metabolites derived from five volatile organic compounds in human urine using on-line solid-phase extraction coupled with liquid chromatography-tandem mass spectrometry. Talanta. 2015;132:469-78.

52. Einig T, Dehnen W. Sensitive determination of the benzene metabolite S-phenylmercapturic acid in urine by high-performance liquid chromatography with fluorescence detection. J Chromatogr A. 1995;697(1-2):371-5.

53. Buratti M, Brambilla G, Fustinoni S, Pellegrino O, Pulviremti S, Colombi A. Determination of monobromobimane derivatives of phenylmercapturic and benzylmercapturic acids in urine by high-performance liquid chromatography and fluorimetry. J Chromatogr B Biomed Sci Appl. 2001;751(2):305-13.

54. Fan R, Wang D, She J. Method development for the simultaneous analysis of trans,trans-muconic acid, 1,2-dihydroxybenzene, S-phenylmercapturic acid and S-benzylmercapturic acid in human urine by liquid chromatography/tandem mass spectrometry. Anal Methods. 2015;7(2):573-80.

55. Tranfo G, Ciadella AM, Paci E, Pigini D. Matrix effect in the determination of occupational biomarkers in urine by HPLC-MS/MS. Prev Today. 2007;3:57-64.

56. Sterz K, Köhler D, Schettgen T, Scherer G. Enrichment and properties of urinary preS-phenylmercapturic acid (pre-SPMA). J Chromatogr B Analyt Technol Biomed Life Sci. 2010;878(27):2502-5.

57. Cocker J, Mason HJ, Warren ND, Cotton RJ. Creatinine adjustment of biological monitoring results. Occup Med (Lond). 2011;61(5):349-53.

58. Borgie M, Garat A, Cazier F, Delbende A, Allorge D, Ledoux F, Courcot D, Shirali P, Dagher Z. Trafficrelated air pollution. A pilot exposure assessment in Beirut, Lebanon. Chemosphere. 2014;96:122-8.

59. Sittert NJ, Boogaard PJ, Beulink GD. Application of the urinary S-phenylmercapturic acid test as a biomarker for low levels of exposure to benzene in industry. Br J Ind Med. 1993;50(5):460-9.

60. Rothman N, Bechtold WE, Yin SN, Dosemeci M, Li GL, Wang YZ, Griffith WC, Smith MT, Hayes RB. Urinary excretion of phenol, catechol, hydroquinone, and muconic acid by workers occupationally exposed to benzene. Occup Environ Med. 1998;55(10):705-11.

61. Qu Q, Melikian AA, Li G, Shore R, Chen L, Cohen B, Yin S, Kagan MR, Li H, Meng M, Jin X, Winnik W, Li Y, Mu R, Li K. Validation of biomarkers in humans exposed to benzene: Urine metabolites. Am J Ind Med. 2000;37(5):522-31.

62. Recommendation from the Scientific Committee on Occupational Exposure Limits for benzene [Internet]. London: Social Europe Journal; 1991. Available from: http://bit.ly/2lZTXeA

63. Lovreglio P, D’Errico MN, Fustinoni S, Drago I, Barbieri A, Sabatini L, Carrieri M, Apostoli P, Soleo L. Biomarkers of internal dose for the assessment of environmental exposure to benzene. J Environ Monit. 2011;13(10):2921-8.

64. Jones K, Mccallum J. Benzene Exposure During Tunnelling - Using Biological Monitoring to Assess Control Measures and Working Practice. Ann Occup Hyg. 2011;55(3):248-52.

65. Andreoli R, Spatari G, Pigini D, Poli D, Banda I, Goldoni M, Riccelli MG, Petyx M, Protano C, Vitali M, Barbaro M, Mutti A. Urinary biomarkers of exposure and of oxidative damage in children exposed to low airborne concentrations of benzene. Environ Res. 2015;142:264-72. 
66. Agência Nacional de Vigilância Sanitária (Brasil). Resolução RE n⿳ 899, de 29 de maio de 2003. Diário Oficial da União 2 jul 2003.

67. Zhang X, Xiong W, Shi L, Hou H, Hu Q. Simultaneous determination of five mercapturic acid derived from volatile organic compounds in human urine by LC-MS/MS and its application to relationship study. J Chromatogr B Analyt Technol Biomed Life Sci. 2014;967:102-9.

68. Paci E, Pigini D, Cialdella AM, Faranda P, Tranfo G. Determination of free and total S-phenylmercapturic acid by HPLC/MS/MS in the biological monitoring of benzene exposure. Biomarkers. 2007;12(2):111-22.

69. Tranfo G, Bartolucci GB, Pigini D, Paci E, Scapellato ML, Doria D, Manno M, Carrieri M. Comparison of hydrolysis and HPLC/ MS/MS procedure with ELISA assay for the determination of S-phenylmercapturic acid as a biomarker of benzene exposure in human urine. J Chromatogr B Analyt Technol Biomed Life Sci. 2010;878(27):2529-33.

70. Ding YS, Blount BC, Valentin-Blasini L, Applewhite HS, Xia Y, Watson CH, Ashley DL. Simultaneous determination of six mercapturic acid metabolites of volatile organic compounds in human urine. Chem Res Toxicol. 2009;22(6):1018-25.

71. Hecht SS, Seow A, Wang M, Wang R, Meng L, Koh WP, Carmella SG, Chen M, Han S, Yu MC, Yuan JM. Elevated levels of volatile organic carcinogen and toxicant biomarkers in Chinese women who regularly cook at home. Cancer Epidemiol Biomarkers Prev. 2010;19(5):1185-92.

72. Iwasaki Y, Sawada T, Hatayama K, Ohyagi A, Tsukuda Y, Namekawa K, Ito R, Saito K, Nakazawa
H. Separation Technique for the Determination of Highly Polar Metabolites in Biological Samples. Metabolites. 2012;2(3):496-515.

73. Li Y, Li AC, Shi H, Junga H, Jiang X, Naidong W, Lauterbach, JH.. Determination of S-phenylmercapturic aci in human urine using an automated sample extraction and fast liquid chromatography-tandem mass spectrometric method. Biomed Chromatogr. 2006;20(6-7):597604.

74. B'Hymer C. Validation of an HPLC-MS-MS method for the determination of urinary benzylmercapturic acid and S-phenylmercapturic acid. J Chromatogr Sci. 2011;49(7):547-53.

75. Liao PC, Li CM, Lin LC, Hung CW, Shih TS. An online automatic sample cleanup system for the quantitative detection of the benzene exposure biomarker S-phenylmercapturic acid in human urine by electrospray ionization tandem mass spectrometry. J Anal Toxicol. 2002;26(4):205-10.

76. Lin LC, Chiung YM, Shih JF, Shih TS, Liao PC. Validation of an online dual-loop cleanup device with an electrospray ionization tandem mass spectrometry-based system for simultaneous quantitative analysis of urinary benzene exposure biomarkers trans, trans-muconic acid and S-phenylmercapturic acid. Anal Chim Acta. 2006;555(1):34-40.

77. Schettgen T, Musiol A, Alt A, Kraus T. Fast determination of urinary S-phenylmercapturic acid (S-PMA) and S-benzylmercapturic acid (S-BMA) by column-switching liquid chromatography-tandem mass spectrometry. J Chromatogr B Analyt Technol Biomed Life Sci. 2008;863(2):283-92. 